\title{
PsySquash: A program for the analysis of PsyScope data files
}

\author{
JONATHAN VAUGHAN, PENNY L. YEE, CHARLES E. HEISTERKAMP, \\ AMY L. GREY, and TIFFANY R. MATTSON \\ Hamilton College, Clinton, New York
}

\begin{abstract}
Many experiment-running programs generate output files that require selection, reduction, and formatting of the raw data before the numbers are suitable for input into statistical packages. PsySquash is a Macintosh program for the selection, organization, and summary of the tabular data that are produced by a widely used freeware data acquisition system, PsyScope. PsySquash serves as a bridge between PsyScope's output data format and the input formats required by common statistical packages such as SAS, SPSS, and SuperAnova. An extension of PsySquash is proposed for use with arbitrary tabular data.
\end{abstract}

PsySquash is a Macintosh computer program that facilitates the selection, organization, and analysis of the data files that are produced by PsyScope and similar experiment-running programs. PsySquash is designed to summarize reaction time and accuracy data across experimental conditions. The authors conceived of PsySquash as a tool for undergraduates who are just learning to manipulate data, as well as for advanced researchers who need a convenient bridge between PsyScope and commercial statistical packages.

Why is a program like PsySquash needed? An experiment-running program such as PsyScope does not automatically produce data that are in the correct format for analysis by existing statistical tools. Furthermore, it is not always possible to predict, at the time at which the experiment program is designed, exactly how the data from that experiment are to be analyzed. In fact, since experimenters are strongly attached to particular statistical analysis packages, it would be inappropriate to confine the users of an experimental program to a particular package. At the same time, general-purpose data manipulation languages such as Microsoft's Excel are expensive and somewhat daunting for novice users such as undergraduate students.

The program code that PsySquash exploits is derived from several sources. The basic analysis part is adapted, with permission, from Squash, written by Earl Hunt, Simon Farr, Colene McKee, and John Palmer. This core was adapted to produce files in the Descriptive Statistics and Compact Variable formats from PsyScope data files by Jonathan Vaughan and Penny Yee. The program was then expanded by Virginia Vaughan to include the Statistics Package format, incorporating the design ideas of Brian MacWhinney, with the support of the PsyScope Consortium at Carnegie Mellon University. T.R.M. was supported by the Howard Hughes Medical Foundation, and A.G., by the Hansmann undergraduate research fund at Hamilton College. We thank Kim Hurme. Brian MacWhinney, and Sidney Perloe for their comments and suggestions. Requests for reprints may be addressed to J. Vaughan, Department of Psychology, Hamilton College, Clinton, NY 13323 (e-mail: jvaughan@hamilton.edu).

\section{OVERVIEW}

\section{PsyScope}

PsyScope is a flexible and powerful program for the generation of perceptual and cognitive experiments. It is capable of presenting auditory and visual stimuli and recording manual and vocal responses. Its uses in the research laboratory (Cohen, MacWhinney, Flatt, \& Provost, 1993) and in the undergraduate cognitive psychology laboratory (Vaughan \& Yee, 1994) have been described previously. The data output file of a typical PsyScope experiment is a chronological, trial-by-trial record of the stimuli and responses of the experiment. Once the data have been acquired, the experimenter has five tasks: (1) to compile the data across subjects by combining their separate data files; (2) to identify the relevant independent variables among the many variables recorded by PsyScope; (3) to select the appropriate dependent variables from the recorded variables; (4) to specify an output format that will be consistent with the next intended step of analysis; and (5) to specify what summary statistics are to be computed.

\section{PsySquash}

PsySquash is a computer program that is intended to facilitate these five steps. An early version was described by Vaughan and Yee (1994), but PsySquash has since undergone several enhancements that have improved its flexibility. The evolution of PsySquash has been guided by several principles:

First, a program for data analysis should be transparent, by exploiting the operating system's graphic user interface to lead the user through the necessary steps of the analysis. The program should, where possible, reinforce the learning of statistical principles by students who are relative novices, by presenting the interface using familiar statistical terms. It should not depend on much specific prior knowledge of its operation. 


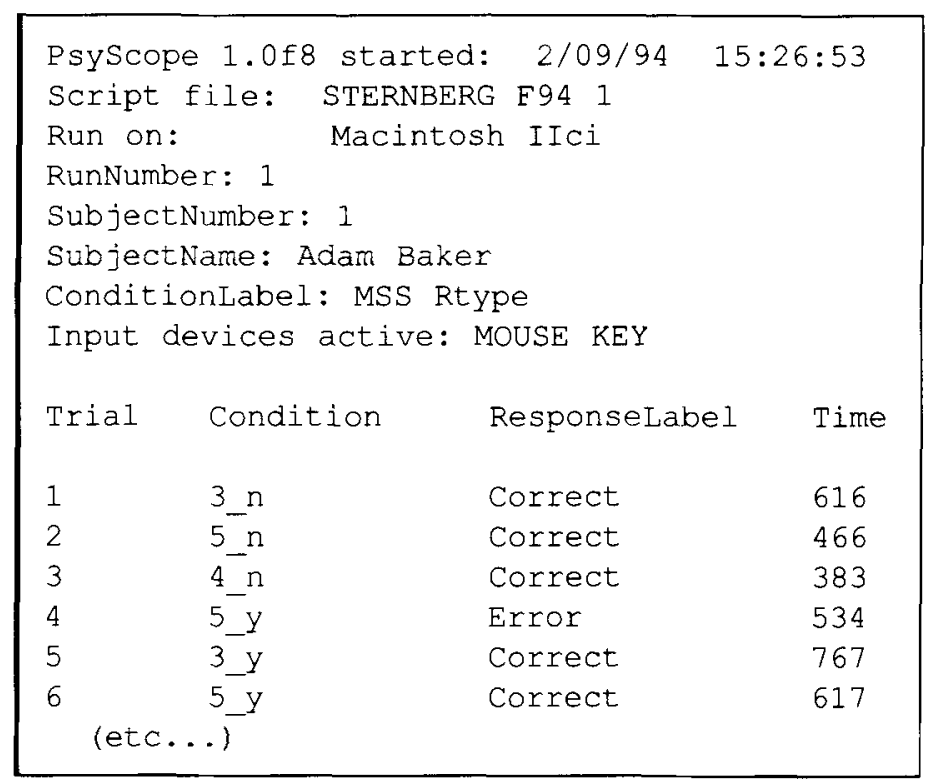

Figure 1. Sample data file produced by PsyScope, edited for clarity. (In this example, the first eight lines are header lines; next, the column label line provides labels for the columns; and the remaining lines are the trial-by-trial data. Note that the ConditionLabel header line provides information that will be used to decode the Condition column of the data file.)

Second, the program should be flexible, for the benefit of not only undergraduates but also researchers. The user may tailor an analysis to a particular purpose-for example, by filtering the data to omit certain values of the independent or dependent variables. For the undergraduate, flexibility has the didactic virtue that the student must think through, and learn to make decisions about, the specifics of each analysis.

Finally, for general utility, the program should be independent of any particular statistical package, or any statistical package at all. PsySquash can generate data in formats compatible with either text-based statistical packages (SAS, SPSS, etc.) or spreadsheet-based ones (SuperAnova, Statview, Excel). Pedagogy is served by having PsySquash also capable of producing descriptive statistics for each subject, if the inferential statistical analysis is to be left to the student.

Let us now turn to a step-by-step description of how PsySquash facilitates the transition between raw data and statistical analysis. First, we need to look at the data format of PsySquash's input files.

\section{PSYSCOPE DATA FILE FORMAT}

A PsyScope experiment typically produces one file for each subject. The data file consists of a variable number of header lines followed by trial-by-trial data in tabular format. Figure 1 shows a sample data file. The first section of a PsyScope data file consists of header lines, in which a session variable and its value appear (separated by the ":" character). The header lines specify a variety of information about the run of the experiment (such as the script used, subject name, etc.). The information in the header lines will be used by PsySquash as it parses the data file to generate some of the variable names that will later guide the experimenter in selecting variables for analysis. Most of these header lines are automatically generated by PsyScope, as specified by the user's experimental script. A special user-generated header line, ConditionLabel, may be used to identify the variables that fall in the Condition column of the data file.

The second part of the example in Figure 1 is a portion (the first six trials) of the trial-by-trial data of one subject's run of the experiment. The columns represent the ordinal trial number, the coded condition of each trial, and the trial outcomes (e.g., accuracy and reaction time).

\section{STEP-BY-STEP ANALYSIS}

The example that follows analyzes an experiment modelled after Sternberg's (1969) memory search paradigm. In this experiment, subjects were shown a string of letters containing up to five letters on each trial, followed by a probe letter. They were then shown a target letter, and they pressed a "yes" key if the letter had been in the memory set, and a "no" key if it had not. Thus, in the example there are two independent variables, memory set size (1-5) and response type ("yes" or "no").

\section{Specification of the Data Files}

When PsySquash is launched, it permits the selection of multiple data files, each of which holds one session or more from a single subject. The data file selection dia$\log$ box permits the selection of individual files, or sets of files chosen with a wild card specification. For example, in Figure 2, all files beginning with "ST" and 


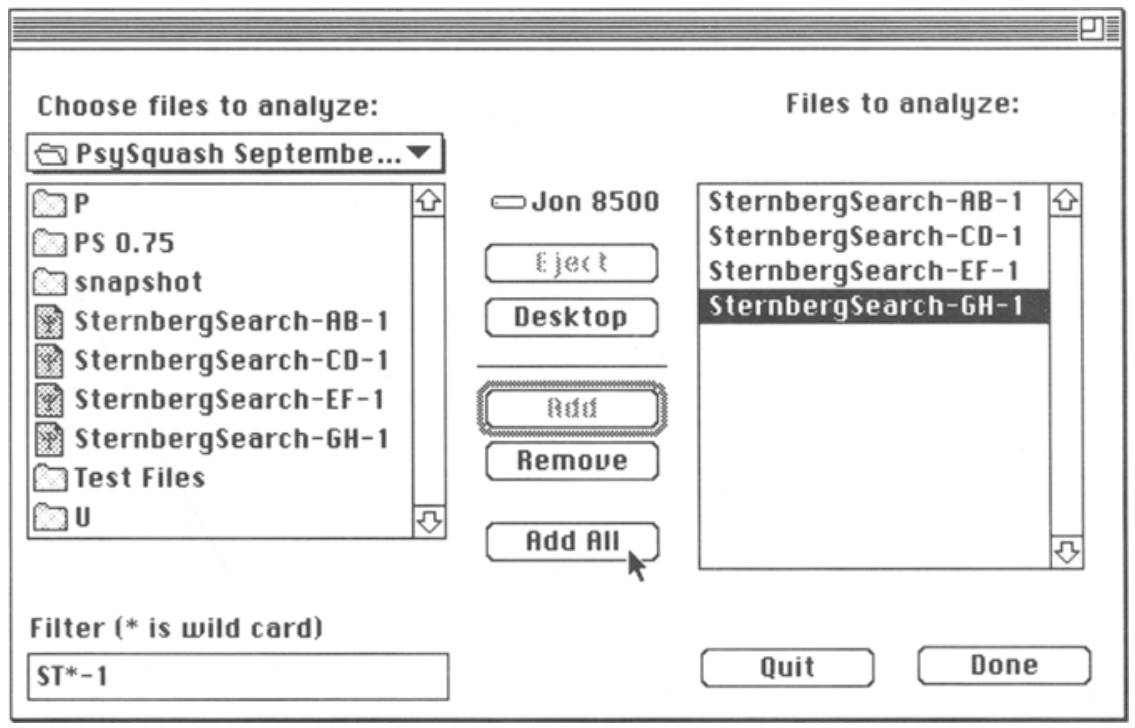

Figure 2. File selection dialog box. (The wild card, $S T^{*}-1$, has been set to select all files that begin with ST and end with -1 .)

ending with " -1 " have been added to the list to be analyzed (the "*" in the filter matches any string of characters between "ST" and " -1 " in the file name). In this example, all of the files selected are in one folder; the user could now switch to another folder and add additional files to the set already selected.

\section{Selection of Statistical Output Format}

PsySquash generates output in one of three different formats: Statistics Package format, trial-by-trial data for input into text-oriented statistical packages (such as SAS and SPSS $^{2}$ in their mainframe incarnations); Descriptive Statistics format, a tabular, subject-by-subject summary of variables so that the experimenter can get an overview of the results; and Compact Variable format, summary statistics of a single dependent variable in spreadsheet format for input into table-oriented statistical packages such as StatView, SuperAnova, ${ }^{3}$ or SAS. For brevity, we will show the output for Descriptive Statistics and Compact Variable formats only (the Statistics Package format

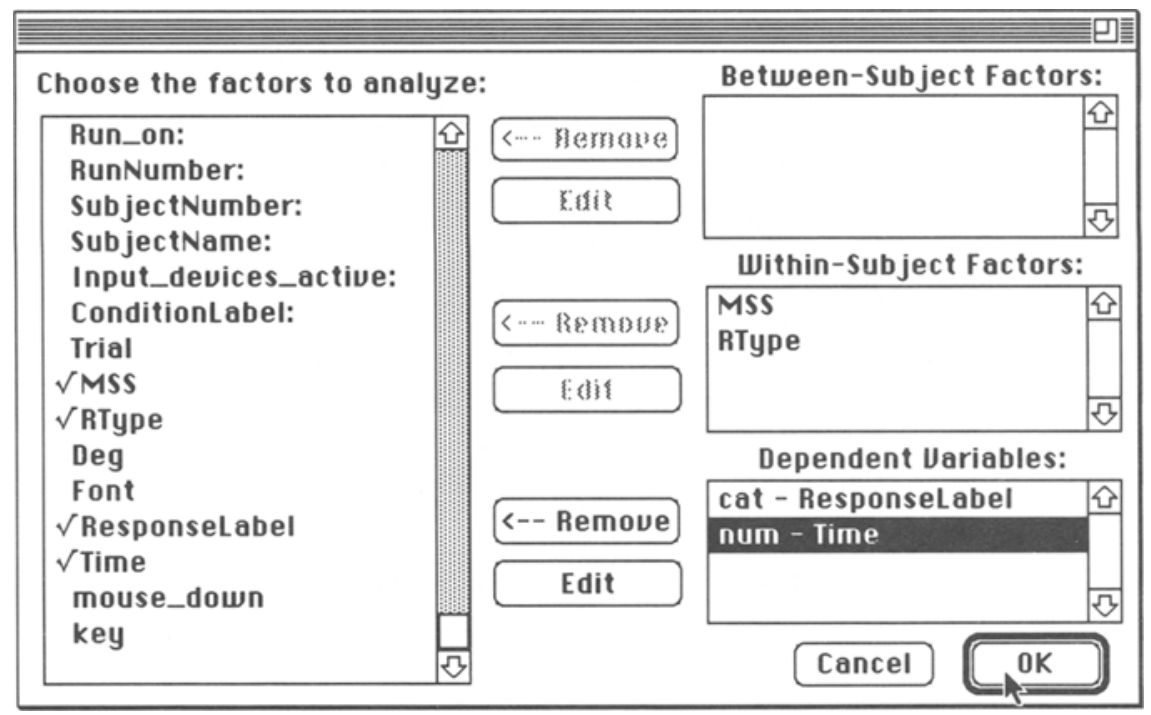

Figure 3. Variable Selection dialog box. (In this example, the selected independent variables are MSS, memory set size, which varies from 1 to 5 , and Rtype, response type, which may take on the values " $y$ " for $y$ es and " $n$ " for no. ResponseLabel, the "correct" or "error" label assigned to each response by PsyScope, is the selected categorical dependent variable, and Time, the reaction time recorded by PsyScope, is the selected numeric dependent variable.) 


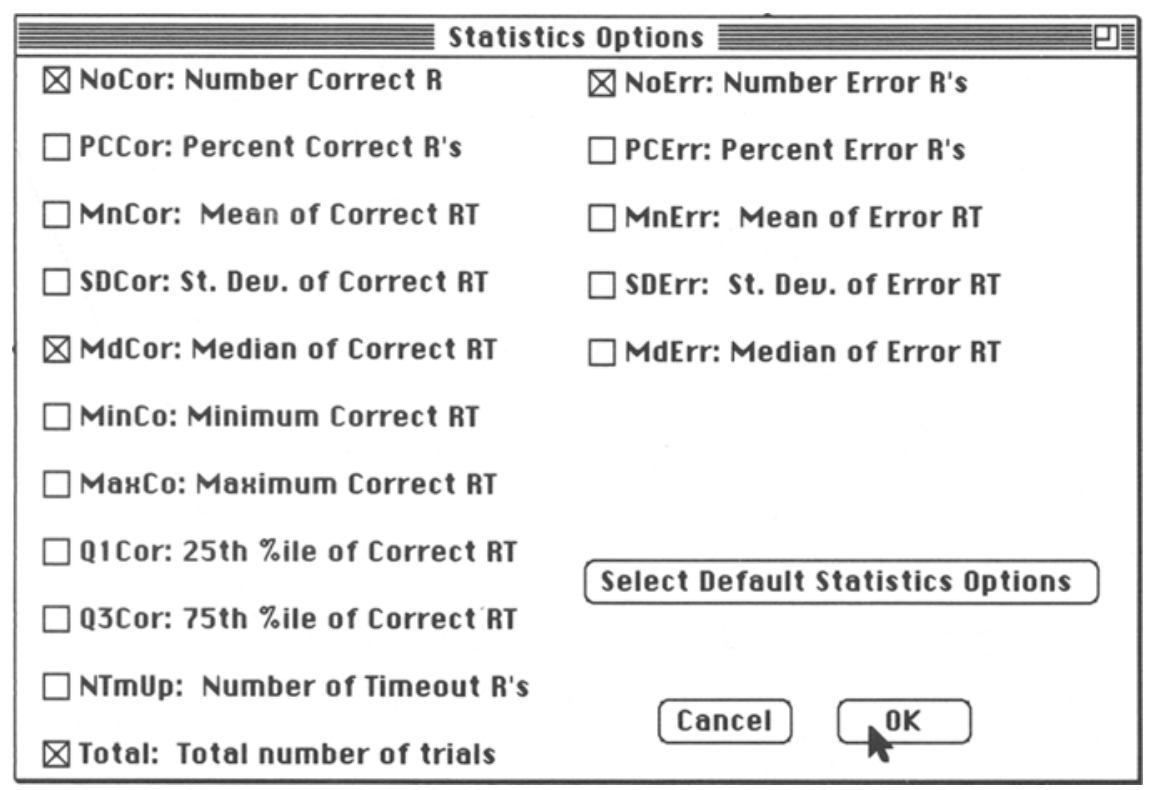

Figure 4. Selection of summary statistics for Descriptive Statistics output.

is described in the full PsySquash documentation; see Availability, below).

\section{Specification of Independent Variables}

The independent variables to be used in an analysis can be specified from those that appeared in the data file (Figure 3), using a point-and-click dialogue. A variable's specification may come from any of several sources: for a between-subjects variable, the file name, the file number, or one of the the header lines of the data file; for a within-subjects variable, the ConditionLabel header line or the column headers of the column label line (the line that begins "Trial. .." in Figure 1). Independent variables may be either numeric or alphabetic.

\section{Specification of Dependent Variables}

Categorical dependent variables (such as ResponseLabel, which may have the values "correct" or "incorrect") and numeric dependent variables are selected similarly.

\section{Selection of Statistics and Data Output File}

When the Descriptive Statistics option has been selected, any desired combination of dependent variables can be selected (Figure 4). Figure 5 shows the computed descriptive statistics (number and median of correct RT's, number of errors, and total number of trials) for two independent variables. Note that there are 2 levels of the first variable (" $n$ " and " $y$ ") and 5 of the second $(1,2$, 3,4 , and 5), corresponding to the memory set sizes and

\begin{tabular}{|ccccccccc|}
\hline Subject & Obs & Rtype & MSS & NoCor MdCor & NoErr & Total \\
1 & 1 & $\mathrm{n}$ & 1 & 8 & 466 & 0 & 8 \\
1 & 2 & $\mathrm{n}$ & 2 & 8 & 483 & 0 & 8 \\
1 & 3 & $\mathrm{n}$ & 3 & 8 & 575 & 0 & 8 \\
1 & 4 & $\mathrm{n}$ & 4 & 8 & 483 & 0 & 8 \\
1 & 5 & $\mathrm{n}$ & 5 & 8 & 483 & 1 & 9 \\
1 & 6 & $\mathrm{y}$ & 1 & 8 & 425 & 0 & 8 \\
1 & 7 & $\mathrm{y}$ & 2 & 7 & 434 & 0 & 7 \\
1 & 8 & $\mathrm{y}$ & 3 & 8 & 483 & 0 & 8 \\
1 & 9 & $\mathrm{y}$ & 4 & 8 & 475 & 2 & 10 \\
1 & 10 & $\mathrm{y}$ & 5 & 8 & 558 & 2 & 10 \\
\hline
\end{tabular}

Figure 5. Descriptive Statistics output. (For brevity, the data for only 1 subject are shown. There are 10 observations for this subject, corresponding to the $2 \times 5$ design of the study. Descriptive statistics were requested for response type, memory set size, number correct, median correct $R T$, number of errors, and total trials.) 


\begin{tabular}{|c|c|c|c|c|c|c|c|c|c|c|}
\hline Sub & n 1 & ก 2 & n 3 & n 4 & ก 5 & y 1 & y 2 & y 3 & y 4 & y 5 \\
\hline 1 & $4 \overline{6} 6$ & $4 \overrightarrow{83}$ & $5 \overline{7} 5$ & $4 \overline{83}$ & $4 \overline{83}$ & $4 \overline{25}$ & $4 \overline{3} 4$ & $4 \overline{83}$ & $4 \overline{7} 5$ & $5 \overline{5} 8$ \\
\hline 2 & 483 & 566 & 558 & 742 & 583 & 450 & 433 & 567 & 533 & 675 \\
\hline 3 & 458 & 533 & 508 & 558 & 692 & 342 & 391 & 558 & 508 & 600 \\
\hline 4 & 424 & 533 & 591 & 575 & 658 & 391 & 433 & 700 & 541 & 625 \\
\hline
\end{tabular}

Figure 6. Compact Variable output (median correct RT) for 4 subjects. (Each row represents the median reaction time of 1 of 4 subjects, broken down by response type and memory set size, as indicated in the column headers. Note that the median correct RTs in the first row correspond to the MdCor column of the Descriptive Statistics output in Figure 6.)

response types. When Compact Variable output has been selected, just one dependent variable may be summarized for further analysis. Figure 6 shows the output of a typical analysis of median correct reaction time.

\section{CONCLUDING REMARKS}

\section{Technical Data: System Requirements and Known Bugs}

PsySquash requires a $680 \times 0$ or Power Macintosh processor and $3 \mathrm{MB}$ of application memory and runs without FPU under Macintosh system 6.0.x or 7.x. PsySquash generates temporary files on disk (e.g., psysquash.data), which may be ignored by the user. Some system extensions that modify the file dialogue, such as the Directory Assistance feature of Norton Utilities for Macintosh, interfere with the use of the filter feature of PsySquash's file-selection dialog box.

\section{Availability and Future Development}

Development of PsySquash is continuing, and the next version is expected to include the ability to summarize generic tabular data in addition to that produced by PsyScope. The most current version of PsySquash is available at no charge for academic users; information and full documentation are available from $<\mathrm{http}$ ://cogito. hamilton.edu $>$. Problems and bug reports are invited, and they may be addressed to jvaughan@hamilton.edu

\section{REFERENCES}

Cohen, J., MacWhinney, B., Flatt, M., \& Provost, J. (1993). PsyScope: An interactive graphic system for designing and controlling experiments in the psychology laboratory using Macintosh computers. Behavior Research Methods, Instruments, \& Computers, 25, 257-271.

STERNBERG, S. (1969). The discovery of processing stages: Extensions of Donders' method. Acta Psychologica, 30, 276-315.

Vaughan, J., \& YeE, P. L. (1994). Using PsyScope for demonstrations and student-designed experiments in cognitive psychology courses. Behavior Research Methods, Instruments, \& Computers, 26, 142-147.

\section{NOTES}

1. SPSS is a product of SPSS, Inc., 444 North Michigan Ave., Chicago, IL 60611.

2. SAS is a product of SAS Institute Inc., SAS Campus Drive, Cary, NC 27513-2414.

3. StatView and SuperAnova are products of Abacus Concepts, 1918 Bonita Ave., Berkeley, CA 94704-1014.

(Manuscript received December 3, 1996; revision accepted for publication February 4, 1997.) 